\title{
Interférence de semelles filantes dans le domaine plastique
}

\author{
Plastic interference of strip footings
}

\author{
C. RYBAK, P. KONDERLA
}

Ecole polytechnique de Wroclaw"

Rev. Franç. Géotech. n 64, pp. 5-9 (juillet 1993)

\section{Résumé}

Cet article présente une étude de l'augmentation de la force portante d'une semelle extrême dans une rangée de semelles parallèles, rugueuses, lorsque l'espacement entre les semelles diminue. Les résultats numériques sont donnés pour un sol sans cohésion, pour trois valeurs: $26^{\circ}, 35^{\circ}, 44^{\circ}$ de l'angle de frottement interne et différentes profondeurs d'encastrement de la semelle extrême. On indique accessoirement le coefficient de la force tangentielle s'exerçant sur une semelle extrême.

\section{Abstract}

The paper deals with the increase in bearing capacity for the rough footings at the end of the row, when the distance between the footings is reduced. Numerical results are presented for cohesionless soils, for 26-, 35-, and 44-degree angle of internal friction. Several founding depths were considered. The coefficient for the tangential force acting on the outermost footing is also investigeted. 


\section{INTRODUCTION}

L'étude concerne une rangée de semelles de fondations parallèles liées entre elles de telle sorte que chacune d'elles est soumise à un déplacement de translation vertical mais sans déplacement horizontal. L'étude actuelle complète les résultats publiés par RYBAK $(1976,1977)$, PULA et RYBAK (1981), pour le cas d'une semelle extrême, pour laquelle les profondeurs d'encastrement de ses côtés ne sont pas égales. Le problème posé est celui de laugmentation de la force portante de cette semelle, lorsque l'espacement entre les semelles diminue.

Pour une semelle isolée, dans le cas d'un sol sans cohésion (sol pulvérulent, $c=0$ ), on pose d'habitude (Terzaghi) :

$$
\mathrm{Q}=\mathrm{B}\left(\mathrm{q} \mathrm{N}_{\mathrm{q}}+0,5 \gamma \mathrm{BN}_{\mathrm{\gamma}}\right)
$$

$\mathrm{Q}$ désignant la charge limite qui peut lui être appliquée, $\mathrm{q}=\gamma \mathrm{D}$ la surcharge due à la profondeur, $\gamma$ le poids volumique du terrain, $\mathrm{N}, \mathrm{N}$ des coefficients sans dimension, dépendant de $\Phi$, langle de frottement interne du sol.

La charge limite peut être calculée aussi selon la formule (RYBAK, 1976):

$$
\mathrm{Q}=\gamma \mathrm{B}^{2} \mathrm{~N}_{\mathrm{q \gamma}}
$$

$\mathrm{N}$ désignant le coefficient sans dimensions,

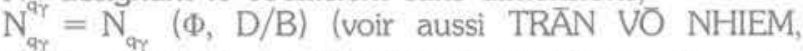
1971; MATAR et SALENÇON, 1979). Les formules (1) et (2) demeurent valables tant que les intervalles libres EL et EP qui séparent une semelle de largeur B des semelles voisines sont plus grands que les longueurs des vagues soulevées à la surface libre, et que les pro. fondeurs d'encastrement DL (du côté gauche) et DP (du côté droit) sont égales. Pour une semelle dans une rangée, dans le cas d'interaction avec les semelles voisines, pour DL $=$ DP on a donné, (RYBAK, 1977 ; PULA et RYBAK, 1981) la formule:

$$
Q^{*}=\gamma B^{2} N^{*}
$$

$\mathrm{N}_{\text {qy }}^{*}$ désignant le coefficient sans dimension,

$\mathrm{N}^{*}{ }_{\text {qry }}=\mathrm{N}_{\text {qu }}^{*}(\Phi, \mathrm{D} / \mathrm{B}, \mathrm{EL} / \mathrm{B}, \mathrm{EP} / \mathrm{B})$. Dans le cas d'une semelle extrême, quand il y a une différence entre les niveaux des terrains DL $\neq$ DP (fig. 1a), on propose la formule:

$$
Q^{D^{*}}=\gamma^{B^{2}} \mathrm{~N}_{Q \gamma}^{D^{*}}
$$

$\mathrm{N}_{\mathrm{q \gamma}}^{\mathrm{D}^{*}}$ désignant le coefficient sans dimension,

$\mathrm{N}_{\mathrm{a \gamma}}^{D^{*}}=\mathrm{N}_{\mathrm{q \gamma}}^{\mathrm{D}^{*}}(\Phi, \mathrm{EP} / \mathrm{B}, \mathrm{DL} / \mathrm{B}, \mathrm{DP} / \mathrm{B})$.

\section{HYPOTHËSES, MÉTHODE DU CALCUL}

Le calcul de la force portante d'une semelle isolée ou en groupe est fait en supposant que:

- le problème est bidimensionel;

- le sol est homogène et isotrope de poids spécifique $\gamma$, et obéit à la loi de plasticité parfaite de Coulomb, d'angle de frottement interne $\Phi$, dans tout le massif;
- l'angle de frottement sol-fondations est égal à $\Phi$ (fondation rugueuse);

- le sol au-dessus du plan horizontal passant par la base de la fondation agit comme une surcharge $\mathrm{q}$, uniforme et normale;

- au-dessous de ce plan l'équilibre limite est atteint dans certaines régions.

Le schéma du calcul de la capacité portante pour une semelle extrême dans une rangée est présenté sur la figure $1 \mathrm{~b}$. Le calcul des contraintes en chaque point des zones en équilibre limite se fait en résolvant le système d'équations aux dérivés partielles de type hyperbolique de SOKOLOVSKY (1960). Dans le schéma du calcul on a supposé l'existence d'un coin rigide solidaire de la base de la fondation, limité par les caractéristiques du champ statique (LUNGREN et MORTENSEN, 1963). Les détails des calculs dans les zones distinctes sont exposés par RYBAK $(1976,1977)$. En utilisant les valeurs des contraintes sur les caractéristiques limitant le coin rigide et, éventuellement, sur la base de la semelle, près des bords, et retranchant le poids du coin rigide on calcule la force portante de la fondation, la force horizontale s'exerçant sur la semelle et l'exentricité de la réaction du sol.

a)

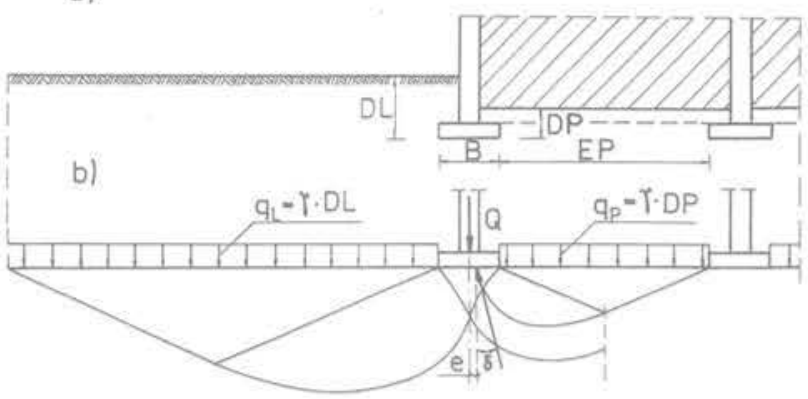

Fig. $1 a$ et $1 b$. - Section montrant de façon visuelle une semelle extrême dans une rangée et schéma d'écoulement.

Fig. $1 \mathrm{a}$ and $7 \mathrm{~b}$ - Cross-section demonstrating the outermost strip foundation and scheme. of the plastic flow.

\section{RÉSULTATS DES CALCULS}

Dans cet article on présente les résultats complets du calcul numérique concernant la force portante d'une semelle extrême, pour trois valeurs: $26^{\circ}, 35^{\circ}, 44^{\circ}$ de l'angle de frottement interne. Pour le calcul de la force portante on donne sur les figures 2 et 3 les valeurs $N_{\text {, }}$ (le cas d'une semelle isolée, DL = DP). La figure 4 donne les rapports de la force portante d'une semelle isolée dans les cas où $D L \geq D P$, et de la force portante d'une semelle isolée dans le cas DL $=$ DP.

Les figures 5,7 et 9 donnent les rapports des $\mathrm{N}_{q \gamma}^{\mathrm{D}^{*}}$ ( $\mathrm{N}_{\text {qy }}^{\mathrm{D}}$ est le coefficient de la force portante dans le cas d'interaction entre les semelles et $\mathrm{DL} \geq \mathrm{DP}$ ). 


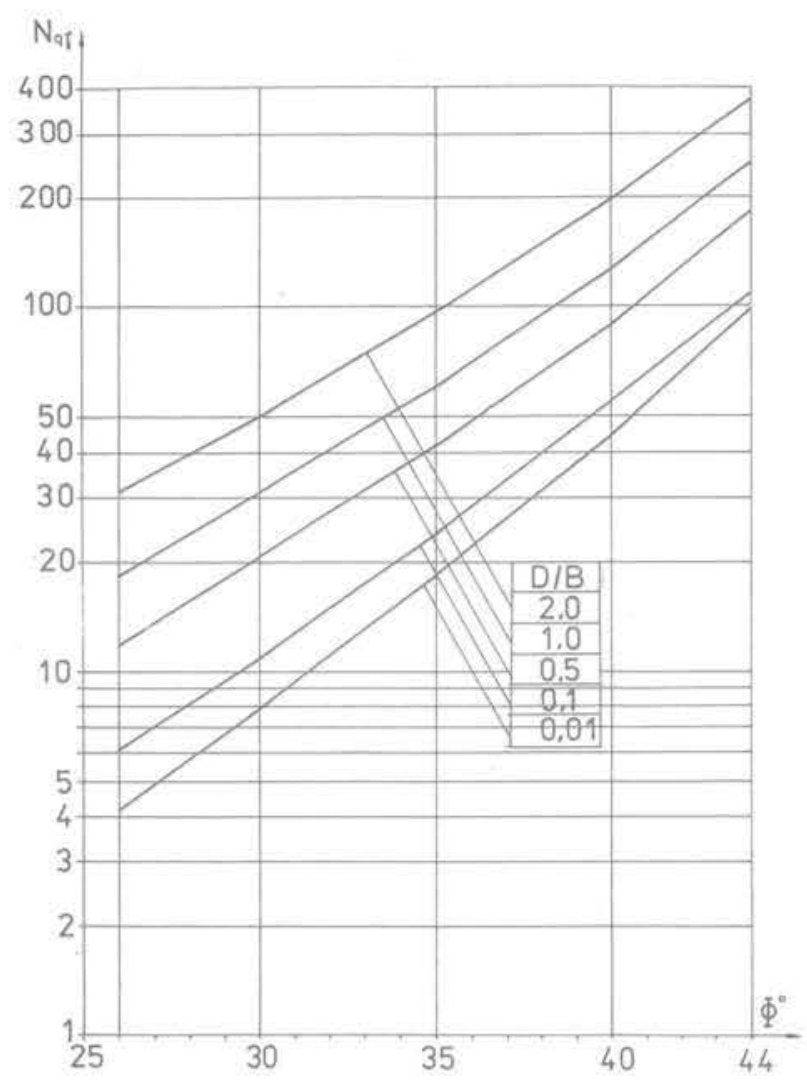

Fig. 2. - Valeurs du coefficient $N_{a_{y}}$ en fonction de $\Phi$ et $\mathrm{D} / \mathrm{B}$.

Fig. 2. - Values of the coefficient $N_{a_{\gamma}}$ as a function of $\Phi$ and $D / B$.

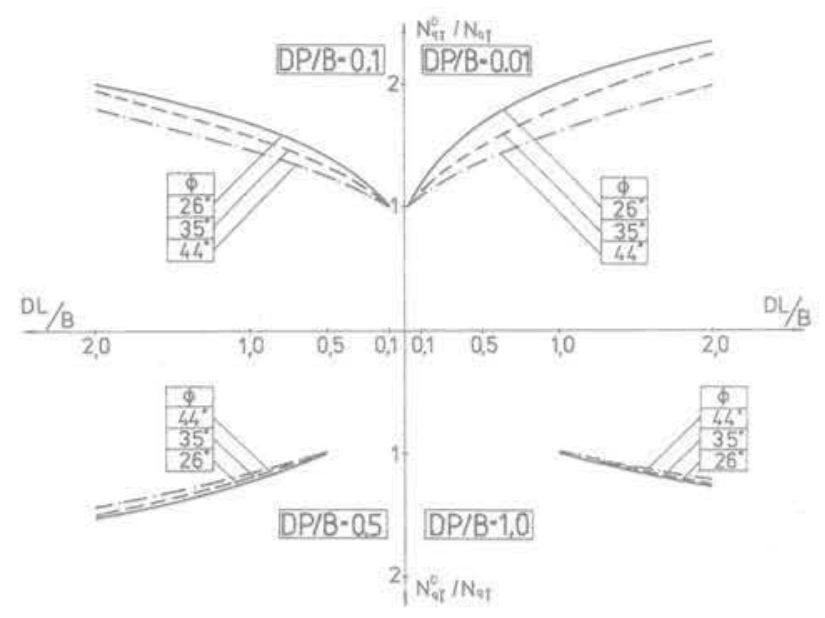

Fig. 4. - Valeurs du rapport $N_{q_{\gamma}}^{D} / N_{Q_{\gamma}}$

Fig. 4. - Values of the coefficients ratio $N_{q_{\gamma}}^{D} / N_{a_{\gamma}}$

Les figures 6,8 et 10 donnent la valeur de $\operatorname{tg} \delta$ coefficient qui exprime le rapport entre la force portante $\mathrm{Q}^{\mathrm{D}}$ et la force tangentielle s'exerçant sur la semelle extrême. Sur les figures 5,7 et 9 on voit que l'augmentation de la capacité portante d'une fondation dans un groupe tend vers l'infini quand l'espacement entre

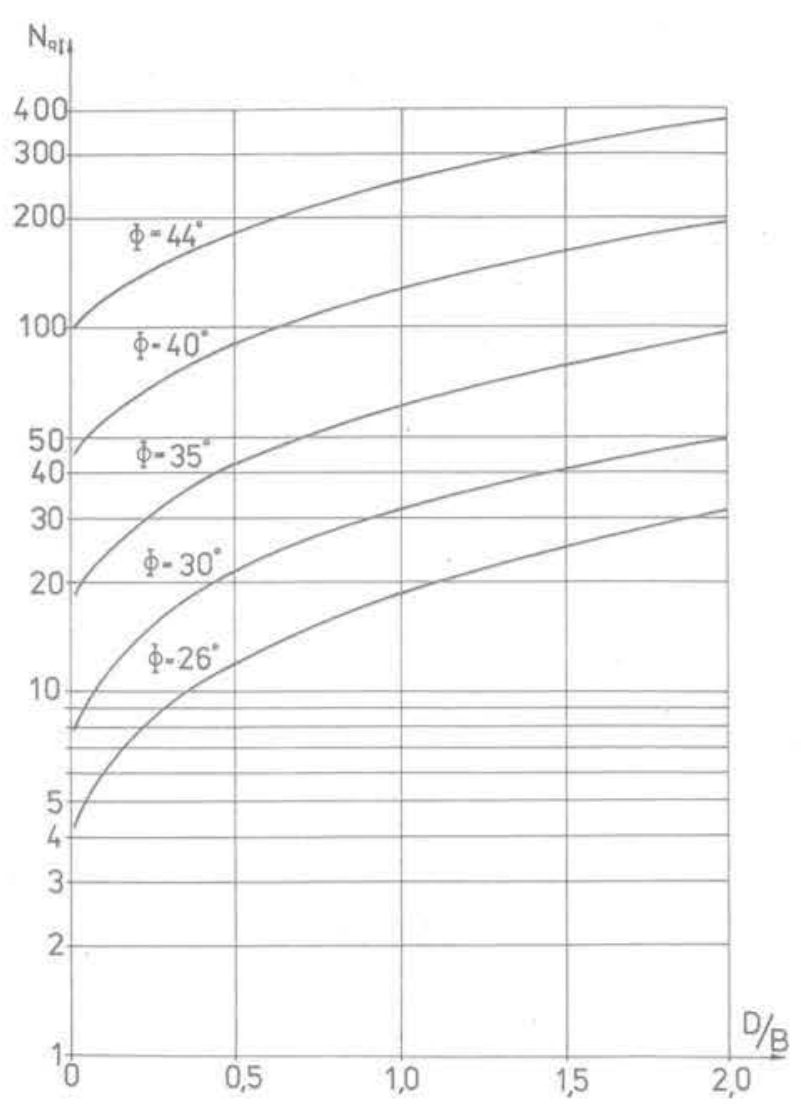

Fig. 3. - Valeurs du coefficient $N_{q_{\gamma}}$ en fonction de D/B et $\Phi$.

Fig. 3. - Values of the coefficient $N_{a_{\gamma}}$ as a function of $D / B$ and $\Phi$.

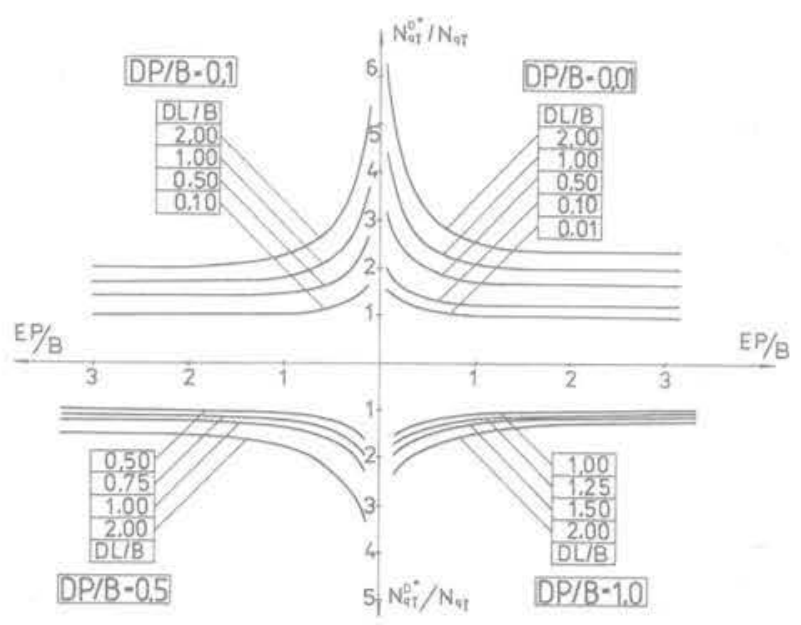

Fig. 5. - Valeurs du rapport $N_{a}^{p} / N_{\gamma}$ pour $\Phi=26^{\circ}$.

Fig. 5. - Values of the coefficients ratio $N_{q_{\gamma}}^{0 .} / N_{a_{\gamma}}$ for $\Phi=26^{\circ}$

les fondations tend vers 0 . En réalité (MANDEL, 1963; RYBAK, 1976, 1977) cette augmentation est limitée, parce que d'autres modes d'écoulement doivent être envisagés, deux ou plusieurs semelles voisines pouvant agir comme une seule, les intervalles entre elles restant en équilibre surabondant. 


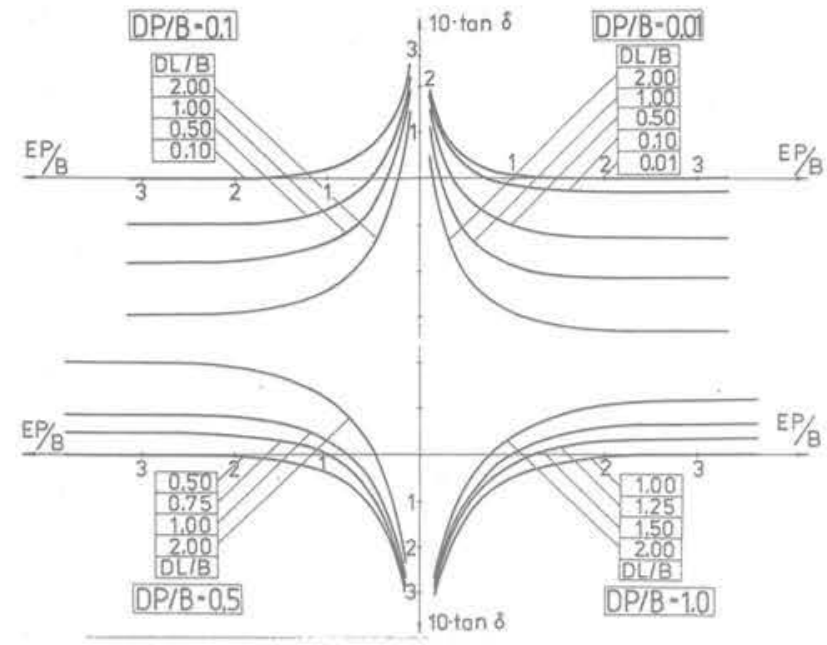

Fig. 6. - Valeurs du coefficient tan $\delta$ pour $\Phi=26^{\circ}$. Fig. 6. - Values of the coefficient tand for $\Phi=26^{\circ}$.

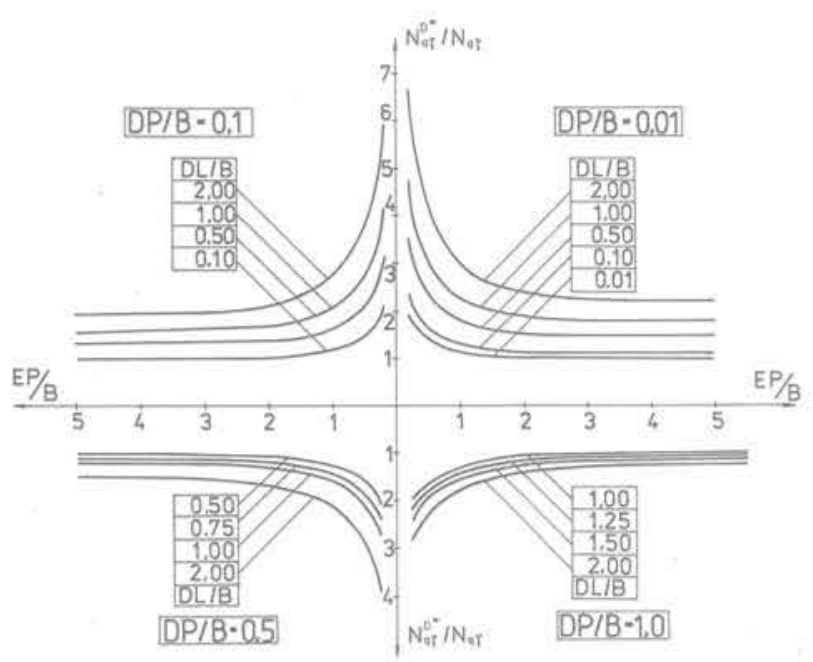

Fig. 7. - Valeurs du rapport $N_{a_{\gamma}}^{D} / N_{a_{y}}$ pour $\Phi=35^{\circ}$.

Fig. 7. - Values of the coefficients ratio $N_{q_{Y}^{*}}^{D^{*}} N_{q_{\gamma}}$ for $\Phi=35^{\circ}$.

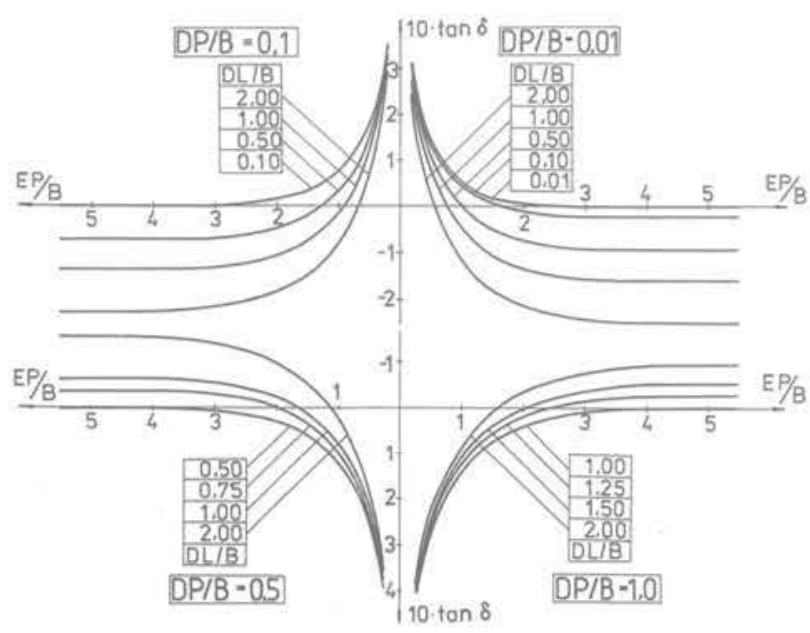

Fig. 8. - Valeurs du coefficient tan $\delta$ pour $\Phi=35^{\circ}$, Fig. 8. Values of the coefficient $\tan \delta$ for $\Phi=35^{\circ}$.

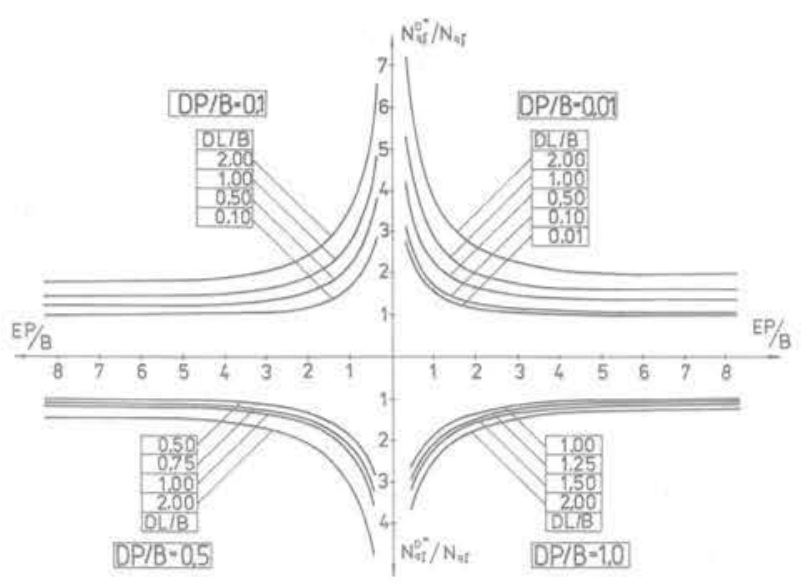

Fig. 9. - Valeurs du rapport $N_{q .}^{D^{\circ}} / N_{q_{\gamma}}$ pour $\Phi=44^{\circ}$.

Fig. 9. - Values of the coefficients ratio $N_{q_{\gamma}^{*}} / N_{Q_{y}}$ for $\Phi=44^{\circ}$

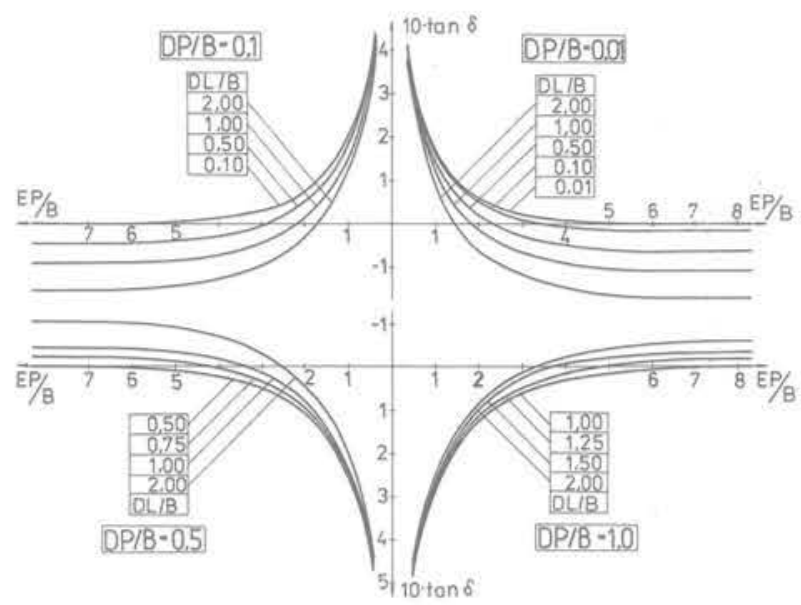

Fig. 10. - Valeurs du coefficient tan $\delta$ pour $\Phi=44^{\circ}$.

Fig. 10. - Values of the coefficient tand for $\Phi=44^{\circ}$.

\section{CONCLUSION}

L'étude précédente montre que l'interaction a un effet positif sur la force portante. Pour une semelle extrême, on observe une influence significative sur la force portante de l'encastrement de la base au-dessous du terrain du côté extérieur d'une rangée de semelles. L'augmentation de la force portante est faible pour des espacements normaux et pour $\Phi$ petit, et est en revanche fort appréciable pour $\Phi>30^{\circ}$. Un autre résultat notable est la valeur de la force tangentielle s'exerçant sur une semelle d'extrêmité.

\section{REMERCIEMENTS}

Les auteurs remercient le professeur J. Kravtchenko de l'Université Joseph-Fourier de Grenoble pour son importante contribution à la réalisation de cette étude. 


\section{BIBLIOGRAPHIE}

GIROUD J.P., TRĀN VO NHIEM, OBIN J.P. (1973), Tables pour le calcul des fondations, Paris, Dunod, tome III.

LUNGREN H., MORTENSEN K. (1953), Determination of the bearing capacity of continuous footings on sand. Comptes rendus du $3^{\circ}$ Congrès international de Mécanique des Sols et des Travaux de Fondation, Zurich, t. 1, p. 409.

MANDEL J. (1963), Interférence plastique de fondations superficielles. Proc. of the International Conference on Soil Mechanics and Foundation Engineering, Akadémiai Kiadó, Budapest, pp. 267. 279.

MANDEL J. (1965), Interférence plastique de semelles filantes. Proc. of the 6th International Conference on Soil Mechanics and Foundation Engineering, Montréal, vol. 2, pp. 127-131.

MATAR M., SALENÇON J. (1979), Capacité portante des semelles filantes. Revue française de Géotechnique $\mathrm{n}^{\circ} 9$, pp. 51-76.
PULA O., RYBAK C. (1981), Sur l'interaction de semelles filantes dans le domaine plastique. Proc. of the Xth International Conference on Soil Mechanics and Foundations Engineering, Stockholm, pp. 235-237.

RYBAK C. (1976), Pouvoir portant du milieu sans cohésion chargé d'une rangée de semelles parallèles. Studia Geotechnica VII, 1-2, Université polytechnique de Wroclaw, Pologne, 1976, pp. 53-63.

RYBAK C. (1977), Interférence de semelles filantes dans le domaine plastique. Studia Geotechnica, VIII, 1-2, Université polytechnique de Wroclaw, Pologne, 1977, pp. 47-55.

SOKOLOVSKI V. (1960), Statics of granular media. London, Pergamon Press.

TRĀN VO NHIEM (1971), Force portante limite de fondations superficielles et résistance maximale à l'arrachement des ancrages. Thèse de Docteuringénieur, Université de Grenoble. 